\title{
Long-term survival after abdominal aortic aneurysm repair
}

\author{
José Manuel Llaneza ${ }^{1}$, Norberto Corral $^{2}$, Sara Busto ${ }^{1}$, Amer Zanabili ${ }^{1}$, \\ and Manuel Alonso ${ }^{1}$
}

\begin{abstract}
In this paper, the 10-year survival rate for elective abdominal aortic aneurysm repair in the Hospital Universitario Central de Asturias is statistically analyzed. It it a retrospective, observational and descriptive study of demographic and clinical variables on patients undergoing either open or endovascular abdominal aortic aneurysm repair with respect to the variable exitus. The study comprises 256 patients who were treated for abdominal aortic aneurysm repair along the period from January 2003 to December 2006. Groups of patients did not show a homogeneous behaviour, since diabetes, old age, cardiac and renal pathologies had a negative effect on their control and monitoring. The average 10-year survival rate amounts $37.9 \%$, cancer being the major cause of mortality (lung cancer being the most frequent one) and cardiovascular diseases were the second major cause of patients dead.
\end{abstract}

\section{Introduction}

Abdominal aortic aneurysm (AAA) is a vascular disease with a prevalence greater than $4 \%$ on the population over 65 years old. This prevalence compels healthcare systems to spend numerous resources to the early diagnosis, the monitoring and the surgical repair of the AAA when required. This is especially critical since AAAs tend naturally to grow, what puts pressure on nearby structures and may eventually rupture with catastrophic consequences. Nowadays, it is well-known that preventing aneurysm rupture by repairing it leads to excellent postoperative survival rates. This type of surgery

\footnotetext{
${ }^{1}$ Servicio de Cirugía Vascular, Hospital Universitario Central de Asturias, Oviedo, Spain, . ${ }^{2}$ Departamento de Estadística, I.O. y D.M., Universidad de Oviedo, Spain, norbert@uniovi.es

Dedicatoria bonita diciendo lo majo que era Pedro
} 
is prophylactically recommended, so to avoid the aneurysm sac rupture, what would substantially increase the perioperative mortality.

Along the last years the anaesthesia and resuscitation techniques have improved. As a consequence, the elective surgery outcomes, both those associated with open AAA repair (OAR) and those associated with endovascular one (EVAR), have also improved. Nevertheless, there are different concomitant risk factors in connection with AAA development so that, in several cases in which the AAA is treated, the survival cannot be reliably predicted.

In the literature on the topic, one can find many monitoring studies allowing us to identify short-term mortality factors for these patients, but longterm ones are very scarce. This paper aims to examine the 10-year survival rate of patients who have been treated with elective AAA in the Hospital Universitario Central de Asturias (HUCA), as well as to statistically analyze both the OAR and the EVAR results.

\section{Materials and methods}

This was a retrospective, observational and descriptive study of the patients who underwent elective surgical repair of AAA in HUCA from January 2003 to December 2006.

As the election criterion in HUCA, patients undergoing AAA repair had a maximum diameter of AAA over $5.5 \mathrm{~cm}$, an enlargement over $5 \mathrm{~mm}$ along the last six months or an enlargement over $1 \mathrm{~cm}$ along the last year. These patients were treated either with OAR or with EVAR, depending on the anatomical characteristics of the AAA, the age or the risk factors for surgery. The study in this paper does not include patients who had an emergency surgery.

The monitoring of these patients was addressed through outpatients periodical appointments of the HUCA Vascular Unit. For OAR patients, the first apointment was scheduled six months after surgery, and later by annual check ups, which included clinical examination and a doppler study and medical image test (usually eco-doppler) every five years thereafter, or whenever it is required due to suspecting the patient having complications. EVAR patients underwent a computerized axial tomography (CAT) with contrast (angioCT) during the first postoperative month, and six months later. In case the EVAR patient was free from endoleak, the monitoring is annual and consisted of an eco-doppler in our vascular lab and a simple abdominal X-ray. In case the EVAR patient showed either (type I and III) high-pressure endoleak or type II endoleak with aneurysm sac enlargement, angio-CT with contrast was considered to treat him/her. Once the problem was solved, monitoring consisted of ecographic controls.

Demographic and clinical analyzed variables were vascular risk factors, lung, cardiac and renal associated pathologies as well as risk scales collected 
in a registry database of all the AAA in the HUCA. To fill in the information about variables exitus (death) and exitus date, the information was drawn from the clinical history of HUCA, the digitalized clinical histories of the public hospitals in the Principality of Asturias, and the mortality records in the Principality.

Causes of exitus were classified into 4 groups, namely, AAA-related death, cardiovascular disease (ICD-10), cancer, and other diseases.

The statistical analysis was carried out by the Department of Statistics, OR and TM in the Faculty of Sciences of the University of Oviedo by using (open access) R packages. Comparisons for qualitative variables were based on chi-square tests. Comparisons for quantitative variables were based on Student $t$ tests. Survival analyses were based on the Kaplan-Meier test. Finally, the inferential analysis about the influence of some of the variables on the mortality considered Cox regression model.

\section{Results}

January 2003 to December 2006, 258 patients underwent elective AAA surgery in HUCA, either by OAR or EVAR. Along these four years the surgical trend experienced a drastic change (see Figure 1). More concretely, whereas in the first year 57 of 74 patients underwent OAR and 17 underwent EVAR, in the fourth year 20 of 57 patients underwent OAR and 37 underwent EVAR. This change can be easily seen in the graphical display in Figure 1. Actually, what one can easily observe by looking at the whole figure is that this trend to consider EVAR more frequently than OAR has been consolidated in the Vascular Surgery Unit of HUCA along the years (to date around 4 out of 5 elective AAA patients undergo EVAR).

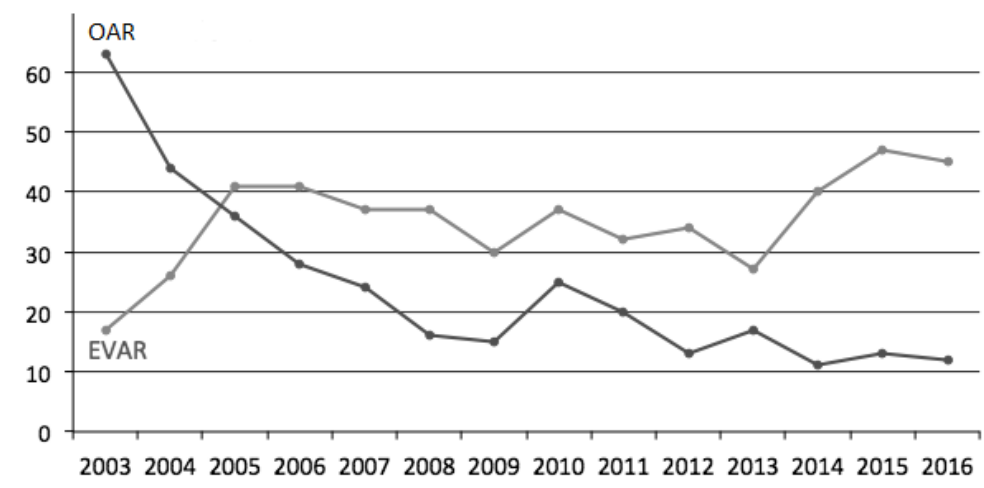

Fig. 1 Evolution of the numbers of patients undergoing OAR and EVAR in the period 2003-2016 in HUCA 
If we constrain to the four-year period 2003-2006, 134 of the 258 treated patients (i.e., 51.94\%) underwent OAR and 124 (i.e., 48.06\%) underwent EVAR. In the second group 2 patients were discarded from the study: one of them was lost to clinical follow-up, and for the other one the endovascular stenting failed so that we cannot consider him as a suitable surgery candidate because of having comorbidity. Concerning the 30-day and in-hospital mortality was 6 (all of them corresponding to OAR) what indicated $4.84 \%$ of immediate mortality in the OAR group and $0 \%$ in the EVAR. As a consequence from these losses, the sample size was reduced to 250, 128 of them belonging to the OAR group and 122 belonging to the EVAR group.

In conducting the univariate analysis the results were gathered in Table 1. One can easily observe that OAR and EVAR lead to significantly different results (i.e., $p$-values are very small) in connection with age, percentage of octogenarian patients, major associated risk factors, cardiopathies, and surgical risk scales (where ASA score corresponds to the American Society of Anaesthesiologists scale, TIA is the acronym for Transient Ischemic Attack, and Glasgow score means the well-known Glasgow aneurysm score).

Table 1 Univariate descriptive and inferential comparative analyses between OAR vs EVAR groups for different demographic and clinical variables

\begin{tabular}{||c||c|c||c||}
\hline \hline Variable & $\begin{array}{c}\text { OAR } \\
(134)\end{array}$ & $\begin{array}{c}\text { EVAR } \\
(122)\end{array}$ & $p$-value \\
\hline \hline Age & $69.25 \pm 7.68$ & $75.75 \pm 7.08$ & .000 \\
\hline Octogenerians & $7(5.2 \%)$ & $42(33.9 \%)$ & .000 \\
\hline Smoking & $125(93 \%)$ & $104(83.9 \%)$ & .017 \\
\hline Dyslipidemia & $71(53 \%)$ & $45(36.3 \%)$ & .007 \\
\hline Cardiopathy & $52(38.8 \%)$ & $68(54.8 \%)$ & .010 \\
\hline ASA scale & 2.54 & 2.73 & .046 \\
\hline TIA & 74.01 & 69.63 & .020 \\
\hline Glasgow score & $74.23 \pm 10.96$ & $82.56 \pm 9.98$ & .000 \\
\hline \hline
\end{tabular}

Due to the significant different behaviour of the two groups, separate (instead of comparative) analyses were performed for OAR and EVAR groups in order to examine the long-term evolution of patients and the predictive mortality factors. It whould be further emphasized that there were not significant differences associated either with the aneurysm size itself or with the sex of the patient (in fact, the sample only includes 8 women, so one cannot reliably draw statistical conclusions concerning sex).

By means of a multivariate logistic regression, for which only the small $p$-values have been gathered in Table 2 , one can conclude that

- for OAR patients, the age, the Diabetes Mellitus, the chronic kidney failure, the level of creatinine in the peripheral blood, the heart failure, the 
atrial fibrillation, the ischemic cardiopathy, and the ASA and Glasgow scores, showed to be predictive factors for mortality;

- for EVAR patients, only the atrial fibrillation, the heart failure and the level of creatinine in the peripheral blood showed to be predictive factors for mortality.

Table 2 Predictive factors of mortality for OAR and EVAR groups

\begin{tabular}{||c|c||c|c||}
\hline \hline \multicolumn{2}{||c||}{ OAR $(134)$} & \multicolumn{2}{c||}{ EVAR $(122)$} \\
\hline predictive factor & $p$-value & predictive factor & $p$-value \\
\hline \hline Age & $<.001$ & Atrial fibrillation & $<.001$ \\
\hline ASA score & .025 & Heart failure & .030 \\
\hline Diabetes Mellitus & .023 & Creatinine & .030 \\
\hline Ischemic cardiopathy & .016 & & \\
\hline Atrial fibrillation & .004 & & \\
\hline Heart failure & .015 & & \\
\hline Kidney disease & $<.001$ & & \\
\hline Creatinine & .008 & & \\
\hline Glasgow score & $<.001$ & & \\
\hline \hline
\end{tabular}

On the other hand, the mortality has been directly analyzed. Early mortality was slightly greater for OAR than for EVAR. In OAR group there were 6 exitus, what means $4.69 \%$ of perioperative mortality, since dead occur within 30 days after surgery. In EVAR group there were 5 exitus, what means $4.10 \%$ of perioperative mortality. However, the situation is reversed afterwards, and this seems to be due to the high mean age and presence of risk factors of patients undergoing EVAR. In fact, many of these patients were included in the EVAR group, because of them being quite old and not fulfilling criteria for OAR, which involved a high surgical risk.

The long-term mortality can be examined on the basis of Figure 2. It can be seen how curves for survival OAR and EVAR (expressed in proportions) distance themselves over the considered 10 years after surgery. In this way, along the 36 months after surgery the distance was up to $5 \%$, whereas dis distance amounted around $20 \%$ at the end of the 10 years after surgery.

If mortality is analyzed for each of the 4 groups associated with the main causes of exitus, conclusions differ from the AAA-related death and other diseases to cancer and cardiovascular diseases.

In connection with the AAA-related death:

- there were no exitus from the OAR group after 10 years, since AAA was rightly excluded and there were no infectious complications; 
- there were 4 exitus from the EVAR group after 10 years, what corresponds to $3.27 \%$; three of the deaths were associated with the rupture of the aneurysmal sac (one of them due to a type II endoleak which did not respond to the treatment of either selective embolizations or the sac, and two of them due to type I endoleaks), and the other one was associated with an endoprosthetic infection.

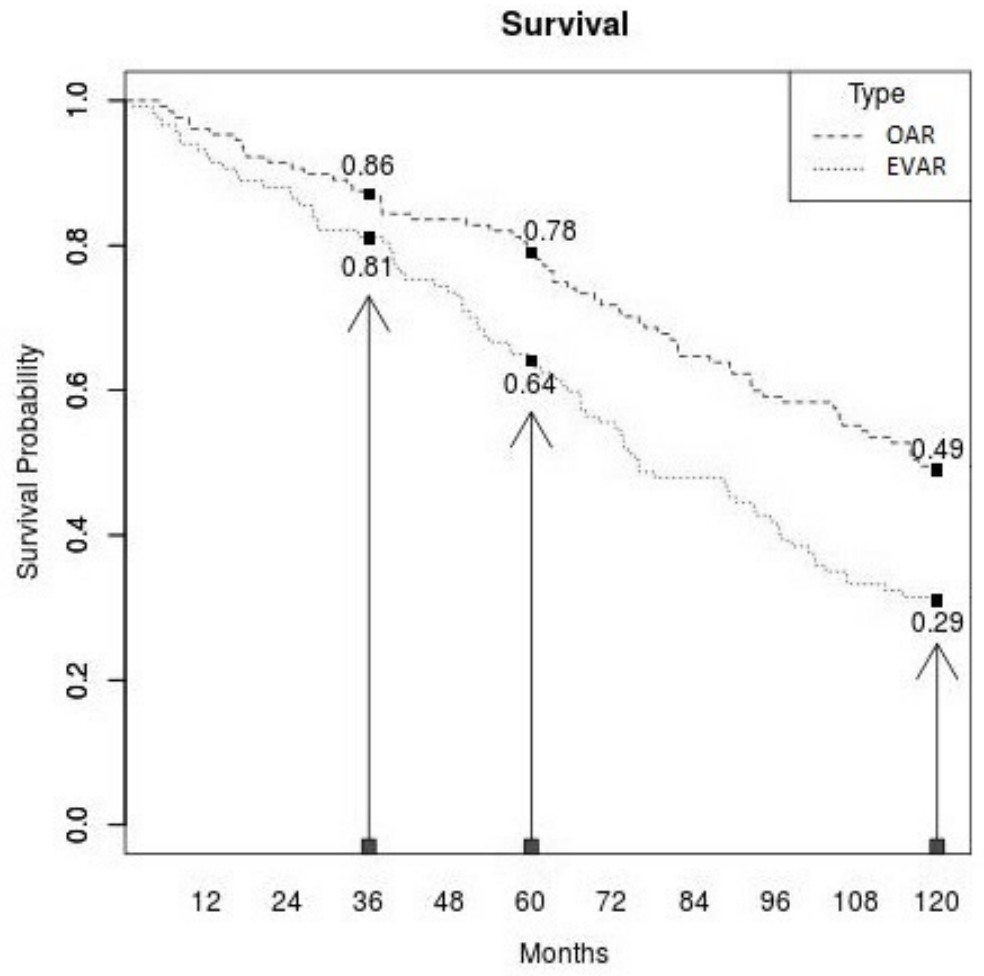

Fig. 2 Long-term (10-year) survival evolution for OAR and EVAR patients in HUCA

Regarding the other diseases group, Figure 3 shows that mortality is more frequent for EVAR than for OAR group. It seems reasonable since EVAR group included a higher comorbidity and more octogenerian patients at the moment of the prosthesis placement.

Among the other two mortality risks groups (cancer and cardiovascular diseases) the 'relative distribution' is quite similar for OAR and EVAR patients, tumor pathology and especially lung cancer prevailing $(56.04 \%$ for OAR and $58.61 \%$ for EVAR) over cardiovascular diseases ( $43.96 \%$ for OAR and $41.39 \%$ for EVAR). This trend was preserved along the follow-up as one can see in the 5-year information gathered in Table 3. 


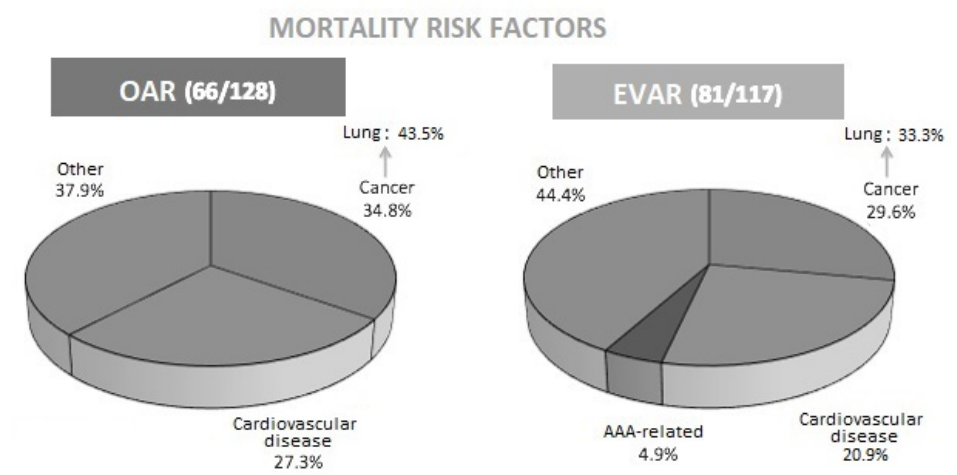

Fig. 3 Long-term (10-year) distribution of the mortality risks in the subsample of exitus for OAR and EVAR in HUCA

Table 3 5-year and 10-year percentages of mortality causes

\begin{tabular}{||c||c|c||c|c||}
\hline \hline \multirow{2}{*}{} & \multicolumn{2}{c||}{ OAR (134) } & \multicolumn{2}{c||}{ EVAR (122) } \\
\cline { 2 - 5 } & $\begin{array}{c}\text { 5-year } \\
\text { percentage }\end{array}$ & $\begin{array}{c}\text { 10-year } \\
\text { percentage }\end{array}$ & $\begin{array}{c}\text { 5-year } \\
\text { percentage }\end{array}$ & $\begin{array}{c}\text { 10-year } \\
\text { percentage }\end{array}$ \\
\hline \hline Cancer & $41 \%$ & $34.8 \%$ & $26.3 \%$ & $29.6 \%$ \\
\hline Cardiovascular disease & $33.1 \%$ & $27.3 \%$ & $21.7 \%$ & $20.9 \%$ \\
\hline Other & $25.9 \%$ & $37.9 \%$ & $39 \%$ & $44.4 \%$ \\
\hline AAA-related & 0 & 0 & $13 \%$ & $4.9 \%$ \\
\hline \hline
\end{tabular}

\section{Discussion}

The early survival of the elective AAA repaired patients has dramatically improved along the last 50 years. The emergence and rapid therapeutic implementation of EVAR has been crucial for such an improvement. This technique allowed vascular specialists to incorporate a relevant cohort of old patients with high comorbidity to undergo AAA repair, in such a way that when studies with age adjustments are performed the improved life expectancy is not lost. These convenient expectations can be found, for instance, in [12] where $70 \% 5$-year survival of the elective OAR is shown which is very similar to our own experience showing $74.6 \%$ 5-year survival.

However, in spite of the technical improvements, mortality in AAA patients is still high, and in some cases it doubles double that of the general population. The main cause for this is a generalized arteriosclerosis with a high rate for stroke, heart attack, and major lower limb amputations due to due to ischaemia, as reported by classical studies like the one in [3]. 
The influence of the arteriosclerosis risk factors is not revealed only in the long-term mortality, but it is well-known that the 30-day mortality is not negligible when we refer to AAA surgery in hospitals conducting a small to moderate number of these surgeries. In all these cases the cardiopathybased mortality is very present, as it can be seen in $[4,11,13]$ where $57.9 \%$ of perioperative mortality after AAA repair is associated with cardiac pathology. Data in this paper are quite similar to those in the last citations, with an important presence of vascular pathology in patients dying, just the second cause for mortality after cancer pathologies. Anyway, ischemic pathology was only influential in the OAR group, whereas arrhythmia by atrial fibrillation and heart failure were always clearly influential in mortality for both groups.

The aneurysm size was considered in the literature to be related to mortality in both the pre-surgery and the perioperative period and to the long-term survival (see, for instance, $[2,14]$ ). One of the long-term mortality theories is the potential direct relationship between the aneurysm size and the arteriosclerosis intensity. Nevertheless, this theory has never been confirmed. Furthermore, one should be aware that aneurysm diameter also depends on other factors, like age, gender, smoking, high blood pressure and high cholesterol level, among others. All this makes that the aneurysm size takes often a rather misleading value, so that it has never been analyzed independently as a risk factor (see $[1,11])$.

In connection with sex, survival studies indicate in general that women have a higher life expectancy than men. However, in case of an underlying aneurysm pathology, mortality of AAA repaired women is equal or greater than mortality of AAA repaired men (see $[7,10,6]$ ). This leads to conclude that the advantage in the general population is reduced so that there is no substantial influence of gender. Our results agree with such an idea of the sex not influencing mortality by AAA repair, although since the number of women in our study is quite small, conclusions are not really reliable.

Patients suffering chronic obstructive pulmonary disease (COPD) have shown worse survival (see $[7,10,15]$ ), especially when they are associated with domiciliary oxygen. In our study COPD was not identified as a risk factor for the long-term mortality, but statistical conclusions are limited in this respect since only a few of our cases were associated with such a permanent oxygen need.

The study in [10] also considers the chronic kidney failure as a factor of bad prognosis. This meta-analysis examines 16 studies, and authors pointed out the limitations caused because of these studies considering different meanings and interpretations of the variable kidney failure. In the study in this paper, kidney failure has only behaved as a risk factor in the OAR group, albeit the level of creatinine in blood has behaved as a mortality risk factor in OAR and EVAR groups.

Other major risk factors, as high blood pressure and Diabetes Mellitus have been also analyzed. The first one was not influential in our study, although in [10] it has been highlighted as a risk factor although by considering 
different diagnoses criteria and treatments; when the left ventricular hypertrophy is added, heterogeneity disappears and the negative influence of the high blood pressure is clear (see $[5,8]$ ). The variable Diabetes Mellitus is not unequivocally defined, in connection with both its diagnosis and its treatment and potential complications, although it seems evident that the longterm mortality tends to increase (see [10]). In case of HUCA, the Diabetes Mellitus has only behaved as a risk factor in the OUR group.

The study in this paper does not consider either socio-economic or ethnic variables which could affect survival in a negative way (see [9]), since our populations seems to be rather homogeneous in these respects.

\section{Conclusions}

The global survival of our data was $37.9 \%$, the percentages of survival being noticeably greater for the OAR, although EVAR patients were definitely older and having a more important associated comorbidity. The major cause of exitus was cancer, especially lung one, for both groups of surgery. The second cause was cardiovascular diseases, and they show in a rather constant way during the follow-up.

Limitations of our study are evident since it concerns a few patients and a short monitoring. Furthermore, the change prompted by the Unit therapeutic choice was clear. Anyway, to continue this type of analyses will be decisive in supporting and suggesting future surgical corrections and the choice of the treatment.

Acknowledgements The research in this paper has been partially supported by the Principality of Asturias/FEDER Grant GRUPIN14-101 and the Spanish Grant MTM201563971-P from the Ministerio de Economía y Competitividad. Its financial support is gratefully acknowledged.

\section{References}

1. Bahia SS, Holt PJE, Jackson D, Patterson BO, Hinchliffe RJ, Thompson MM, Karthilkesalingam A (2015) Systematic review and meta-analysis of long-term survival after elective infrarenal abdominal aortic aneurysm repair 1969-2011: 5 year survival remains por despite advances in medical care and treatment strategies. Eur J Vasc Endovasc Surg 50:320-330

2. Brady AR, Fowkes FG, Thompson SG, Powell JT (2001) Aortic aneurysm diameter and risk of cardiovascular mortality. Arterioscler Thromb Vasc Biol 21:1203-1207

3. Brown OW, Hollier LH, Pairlorelo PC, Kazmier FJ, McCready RA (1981) Abdominal aortic aneurysm and coronary artery disease. Arch Surg 116:1484-1488

4. Conrad MF, Crawford RS, pedraza JD, Brewster DC, LaMuraglia M (2007) Longterm durability of open abdominal aortic aneurysm repair. J Vasc Surg 46(4):669-675 
5. Galinaes EL, Reynolds S, Dombrovskiy VY, Vogel TR (2015) The impact of preoperative statin therapy on open and endovascular abdominal aortic aneurysm repair outcomes. Vascular 23(4):344-349

6. Gloviczki P, Huang Y, Oderich GS, Duncan AA, Kaira M, Fleming MD (2015) Clincal presentation, comorbidities, and age but nor female gender prodict survival after endovascular repair of abdominal aortic aneurysm. J Vasc Surg 61:853-863

7. Grootenboer N, Myriam Hunink MG, Hendriks JM, van Sambeek MRHM, Buth J (2013) Sex differences in 30 days and 5 year outcomes after endovascular repair of abdominal aortic aneurysms in the EUROSTAR study. J Vasc Surg 58(1):42-49

8. Hertzer NR, Mascha EJ (2005) A personal experience with factors influencing survival after elective open repair of ifrarenal aortic aneurysms. J Vasc Surg 42(5):898-905

9. Khashram M, Pitama S, William JA, Jones GT, Roake JA (2017) Survival disparity following abdominal aortic aneurysm repair highlights inequaliti in ethnic and socio-economic status. Eur J Vasc Endovascuc Surg (in press, doi:10.1016/j.ejvs.2017.08.018)

10. Khashram M, William JA, Hider PN, Jones GT, Roake JA (2016) Systematic review and meta-analysis of factors influencing survival following abdominal aneurysm repair. Eur J Endovasc Surg 51:203-215

11. Kertai MD, Boersma E, Westerhout CT, van Domburg R, Klein J, Bax JJ (2004) Association between long-term statin use and mortality afther sucessful abdominal aortic aneurysm surgery. Am J Med 116(2):96-103

12. Mani K, Björck M, Lundkvist J, Wanhainen A (2009) Improved long-term survival after abdominal aortic aneurysm repair. Circulation 120:201-11

13. Norman PE, Semmens JB, Lawrence-Brown MM, Holman CD (1998) Long-term relative survival after surgery for abdominal aortic aneurysm in Western Australia: population based study. BMJ 317:852-856

14. United Kingdom Small Aneurysm Trial Participants, Powell JT, Brady AR, Brown LC, Fowkes FG, Greenhalgh RM, Ruckley CV, Thompson SG (2002) Long-term outcomes of inmediate repair compared with surveillance of small abdominal aortic aneurysm. N Engl J Med 346:1445-1452

15. Von Elm E, Atman D, Egger M, Pocock SJ, Gotzsche PC, Vandenbroucke JP (2007) Strengthening the reporting of observational studies in epidemiology (STROBE) statement: guidelines for reporting osbervational studies. Br Med J 335(7624):806-808 